\title{
Ficolins Promote Fungal Clearance in vivo and Modulate the Inflammatory Cytokine Response in Host Defense against Aspergillus fumigatus
}

\author{
Ninette Genster ${ }^{\mathrm{a}}$ Elisabeth Præstekjær Cramer ${ }^{\mathrm{b}} \quad$ Anne Rosbjerg $^{\mathrm{a}}$ \\ Katrine Pilely ${ }^{a}$ Jack Bernard Cowland ${ }^{b}$ Peter Garred ${ }^{a}$ \\ a Laboratory of Molecular Medicine, Department of Clinical Immunology, Rigshospitalet, Faculty of Health \\ and Medical Sciences, University of Copenhagen, and ${ }^{b}$ The Granulocyte Research Laboratory, Department of \\ Hematology, Copenhagen University Hospital, Copenhagen, Denmark
}

\section{Key Words}

Ficolins · Lectin pathway · Opsonization · Knockout model

\begin{abstract}
Aspergillus fumigatus is an opportunistic fungal pathogen that causes severe invasive infections in immunocompromised patients. Innate immunity plays a major role in protection against $A$. fumigatus. The ficolins are a family of soluble pattern recognition receptors that are capable of activating the lectin pathway of complement. Previous in vitro studies reported that ficolins bind to $A$. fumigatus, but their part in host defense against fungal infections in vivo is unknown. In this study, we used ficolin-deficient mice to investigate the role of ficolins during lung infection with A. fumigatus. Ficolin knockout mice showed significantly higher fungal loads in the lungs $24 \mathrm{~h}$ postinfection compared to wild-type mice. The delayed clearance of $A$. fumigatus in ficolin knockout mice could not be attributed to a compromised recruitment of inflammatory cells. However, it was revealed that ficolin knockout mice exhibited a decreased production of proinflammatory cytokines in the lungs compared to wild-type mice following A. fumigatus infection. The impaired clearance and cytokine production in ficolin knockout mice was
\end{abstract}

independent of complement, as shown by equivalent levels of A. fumigatus-mediated complement activation in ficolin knockout mice and wild-type mice. In conclusion, this study demonstrates that ficolins are important in initial innate host defense against $A$. fumigatus infections in vivo.

(C) 2016 S. Karger AG, Basel

\section{Introduction}

The mold Aspergillus fumigatus is an opportunistic fungal pathogen and one of the leading causes of invasive fungal infections [1]. Invasive pulmonary aspergillosis, a disease of high morbidity and mortality which affects immunocompromised individuals, is of growing concern due to the increased use of immunosuppressive drugs (e.g. for organ transplantation) and chemotherapy treatments [2]. Despite the availability of newer antifungal agents, the prevention and treatment of invasive pulmonary aspergillosis remain problematic [1,2]. It is essential to elucidate the contribution of innate and adaptive immune responses to A. fumigatus to understand the development of invasive disease and to design targeted immunotherapy and vaccination against the fungus.

\section{KARGER}

(C) 2016 S. Karger AG, Basel

$1662-811 X / 16 / 0086-0579 \$ 39.50 / 0$

E-Mail karger@karger.com

www.karger.com/jin
Dr. Peter Garred

Laboratory of Molecular Medicine, Department of Clinical Immunology

Section 7631, Rigshospitalet

Ole Maaloesvej 26, DK-2200 Copenhagen (Denmark)

E-Mail garred@post5.tele.dk 
A. fumigatus is a saprotroph widespread in nature that produces numerous airborne spores known as conidia. Innate immunity plays a major role in protection against A. fumigatus. In immunocompetent hosts, anatomical barriers and professional phagocytes prevent disease when conidia are inhaled on a daily basis [3]. In immunocompromised individuals, however, inhaled conidia germinate and form invasive hyphae which can penetrate pulmonary tissues to cause disease. Upon inhalation of A. fumigatus conidia into the lungs, initial recognition is managed by alveolar macrophages which lead to intracellular degradation of the conidia and secretion of proinflammatory mediators which further recruit neutrophils to assist in fungal clearance [3]. The immune response to A. fumigatus is highly dependent on the recognition of fungal-specific structures, such as cell wall components, by pattern-recognition receptors. Several classes of cellassociated or soluble pattern-recognition receptors are involved in the initial recognition of conidia, including Toll-like receptors [4-7], C-type lectin receptors [8], collectins $[9,10]$, and pentraxins [11], which facilitate the interaction of phagocytes with fungi.

Ficolins are a family of soluble pattern-recognition receptors that activate the lectin pathway of complement similar to mannose-binding lectin (MBL) [12-14]. They comprise a collagen-like domain and a fibrinogen-like domain, of which the latter is involved in ligand recognition. Binding of ficolin to target structures on the pathogen surface activates associated serine proteases which results in the cleavage of downstream complement components $\mathrm{C} 4$ and $\mathrm{C} 2$ and formation of the $\mathrm{C} 3$ and $\mathrm{C} 5$ convertases. This cascade of events leads to the formation of cleavage products that function in opsonization, cell lysis, and the generation of an inflammatory response [15]. In humans, three ficolins have been identified (ficolin-1/ M-ficolin, ficolin-2/L-ficolin and ficolin-3/H-ficolin), whereas in mice two ficolins have been identified (ficolinA and ficolin-B) [13]. Human and murine ficolins are believed to be closely related based on their phylogenetic and biochemical properties [16]. Ficolin-A is regarded as the murine orthologue of ficolin-2, while ficolin- $B$ is the orthologue of ficolin-1. The third ficolin gene exists as a pseudogene in mice.

Research over the past decade has revealed that ficolins recognize a wide range of pathogens [17-20], and that deficiency or low levels of human ficolins is associated with specific infectious diseases [21-23]. In addition, in vivo studies have demonstrated a protective effect of ficolins in murine models of bacterial and viral infections [20, 24-26]. While in vitro studies suggest that ficolins par- ticipate in the elimination of A. fumigatus [27-31], the in vivo role of ficolins during fungal infection has not yet been addressed. Therefore, we used ficolin-deficient mice to elucidate the role of ficolins in host defense against A. fumigatus infection in the present study.

\section{Materials and Methods}

\section{Mice}

Ficolin-A deficient $\left(\mathrm{FCna}^{-/-}\right)$and ficolin-B-deficient $\left(\mathrm{FCnb}^{-/-}\right)$ mice raised on a $\mathrm{C} 57 \mathrm{BL} / 6 \mathrm{~J}$ background were kindly provided by Dr. Endo of Fukushima University, Japan [24], and further backcrossed to C57BL/6J for five generations in our laboratory. From these two mouse lineages, a ficolin double knockout (KO) lineage was generated. Because Fcna and Fcnb are located close together on the same chromosome, we initially crossed $\mathrm{FCna}^{-/-} b^{+/+}$(F5) and $\mathrm{Fcna}^{+/+} b^{+/-}$(N5) mice to obtain double heterozygous offspring that were crossed with $\mathrm{FCna}^{-/-} b^{+/+}$mice to generate a double heterozygous mouse carrying the haplotype $\mathrm{F}_{\mathrm{Cn}} \mathrm{a}^{-} b^{-}$. The obtained mouse carrying genotype $\mathrm{Fcna}^{-} b^{+} / a^{-} b^{-}$was subsequently crossed with a C57BL/6J mouse to generate double heterozygous offspring carrying the haplotype $F \mathrm{Cna}^{-} b^{-}$, and eventually these were interbred. The resulting offspring $\left(\mathrm{FCna}^{-/-} b^{-/-}\right.$mice and their wild-type, WT, littermates) were set up for homozygous breeding; the mice used throughout this study belong to the F2-F4 generations. Female mice were used at 10-12 weeks of age. The mice were housed in pathogen-free, temperature-controlled and air-conditioned facilities with a 12-hour light/dark cycle. Breeding as well as all animal experiments were conducted at the Department of Experimental Medicine, University of Copenhagen, and National University Hospital, Rigshospitalet, Copenhagen, Denmark. All animal experiments were approved by the Danish Animal Experiments Inspectorate (permission No. 2014-15-2934-996) and conducted according to the national Animal Experimentation Act (LBK No. 474 from May 15, 2014). The review board at the Faculty of Health and Medical Sciences, University of Copenhagen, approved this study (P14-122).

\section{Infection}

The A. fumigatus strain was obtained from a clinical isolate 6871 (kindly provided by Prof. Luigina Romani, University of Perugia, Italy) and grown on Sabouraud dextrose agar (SDA; Oxoid) for 4 days at $37^{\circ} \mathrm{C}$. Conidia were harvested by washing plates with sterile phosphate-buffered saline (PBS; $\mathrm{pH} 7.4$ ) supplemented with $0.025 \%$ Tween 80 (PBS-T). The suspension was filtered through a $40-\mu \mathrm{m}$ cell strainer (BD Biosciences) to separate conidia from the contaminating mycelium and washed three times in PBS-T before resuspension in sterile $\mathrm{PBS}$. The absence of mycelium in filtrate was verified microscopically when conidia were counted in a hemocytometer and the concentration was adjusted in sterile PBS. The conidial preparations were stored at $4^{\circ} \mathrm{C}$ for up to 4 months. The conidia viability of the challenge inoculum was confirmed by plating serial dilutions on SDA plates.

Age- and weight-matched mice were anesthetized with isoflurane and a sublethal inoculum dose of $2 \times 10^{7}$ of A. fumigatus conidia in a $50-\mu$ PBS suspension was placed on the nostrils for inhalation. Control mice received $50 \mu \mathrm{l}$ of sterile PBS. After inocula- 
tion, all animals fully recovered from the procedure within $30 \mathrm{~min}$. The clinical appearance and body weight of the animals were monitored daily. A pilot study was carried out to determine the optimal dose (i.e. nonlethal with mild clinical signs of disease and weight loss) for challenge.

\section{Quantification of the Fungal Burden}

The fungal burden in the lungs was determined by quantitative colony-forming unit (CFU) assay. At selected time points postinfection (p.i.), mice were sacrificed by cervical dislocation and the lungs and spleens were aseptically removed. The wet mass of the lungs was determined using a precision balance before the tissue specimens were homogenized in $2 \mathrm{ml}$ of sterile PBS for $1 \mathrm{~min}$ using a mechanical homogenizer (TissueRuptor; Qiagen). Primary homogenates were cultured by serial dilution, plated in triplicate on SDA plates, incubated at $37^{\circ} \mathrm{C}$ for $24 \mathrm{~h}$, and the number of $\mathrm{A}$. $\mathrm{fu}$ migatus colonies was counted. The results are expressed as CFU per lung.

\section{Lung Myeloperoxidase Assay}

Mice were sacrificed by cervical dislocation $24 \mathrm{~h}$ p.i. and the lungs were removed and snap-frozen on dry ice. Lysis buffer $(200$ $\mathrm{mM} \mathrm{NaCl}, 5 \mathrm{~mm}$ EDTA, $10 \mathrm{~mm}$ Tris, 10\% glycine, $1 \mathrm{~mm}$ PMSF, $1 \mu \mathrm{g} / \mathrm{ml}$ leupeptin and $28 \mu \mathrm{g} / \mathrm{ml}$ aprotinin, $\mathrm{pH} 7.4$ ) was added to the lung tissue before homogenization on ice using the TissueRuptor (Qiagen) and the supernatant was immediately frozen and stored at $-80^{\circ} \mathrm{C}$ until analysis. Samples were diluted $500 \times$ and assayed in duplicates by a mouse myeloperoxidase (MPO) ELISA (HK210; Hycult Biotech) following the manufacturer's recommendations.

\section{Cytokine Gene Expression}

Mice were sacrificed by cervical dislocation $24 \mathrm{~h}$ p.i. and the lungs were removed and stored in RNAlater (Applied Biosystems). Lung tissue was homogenized with a mechanical homogenizer (TissueRuptor; Qiagen) and RNA was extracted using the Maxwell 16 tissue LEV Total RNA Purification Kit (Promega) following the manufacturer's recommendations. Total RNA was reverse transcribed with the MuLV reverse transcriptase (Applied Biosystems No. N808-0018) and cDNA was quantified using a Qubit Fluorometer (Invitrogen). Gene expression was assessed by quantitative real-time PCR with TaqMan probes specific for mouse TBP (mM00446971_m1), B2M (Mm00437762_m1), IL-1 $\beta$ (Mm0434228_m1), IL-6 (Mm00446190_m1), and TNF- $\alpha$ (Mm00443258_m1) obtained from Applied Biosystems. Quantitative real-time PCR was performed in triplicate loading $20 \mathrm{ng} / \mu \mathrm{l}$ (TBP and IL-6) or $10 \mathrm{ng} / \mu \mathrm{l}$ (B2M, IL-1 $\beta$ and TNF- $\alpha$ ) of cDNA per reaction and carried out on the Stratagene Mx3005P real-time PCR system (Agilent Technologies). Reaction conditions included incubation at $50^{\circ} \mathrm{C}$ for $2 \mathrm{~min}$ and $95^{\circ} \mathrm{C}$ for $10 \mathrm{~min}$ followed by 40 cycles at $95^{\circ} \mathrm{C}$ for $15 \mathrm{~s}$ and $60^{\circ} \mathrm{C}$ for $1 \mathrm{~min}$. PCR runs included notemplate controls and no-reverse transcriptase controls. The target genes were normalized to a housekeeping gene, TBP or B2M, and results were expressed as fold changes from PBS mock-infected WT mice using the delta-delta Ct method.

\section{Cytokine ELISA Assays}

Mice were euthanized $24 \mathrm{~h}$ p.i. by intraperitoneal injection of pentobarbital before the trachea was exposed through a midline incision. The trachea was cannulated with a sterile 22 -gauge catheter (BD Biosciences) and bronchoalveolar lavage (BAL) was per- formed by instilling and withdrawing $1 \mathrm{ml}$ of cold sterile PBS five times. The BAL fluid (BALF) was centrifuged and the supernatant was removed, immediately frozen and stored at $-80^{\circ} \mathrm{C}$. In separate experiments, serum was obtained from submandibular blood collection from live animals $24 \mathrm{~h}$ p.i. The protein levels of IL- $1 \beta$, IL-6 and TNF- $\alpha$ in BALF and serum were measured in duplicates by mouse cytokine-specific Quantikine ELISA kits (R\&D Systems) according to the manufacturer's recommendations.

\section{Histology and Immunohistochemistry}

Mice were euthanized $24 \mathrm{~h}$ p.i. by intraperitoneal injection of pentobarbital before the trachea was exposed through a midline incision. The trachea was cannulated with a sterile 22 -gauge catheter (BD Biosciences) and the lungs were expanded with $1 \mathrm{ml}$ of $10 \%$ neutral-buffered formalin. Lungs were fixed for $24 \mathrm{~h}$ and stored in $70 \%$ ethanol prior to paraffin embedding. For histology, tissue sections of $5 \mu \mathrm{m}$ were stained with hematoxylin and eosin (HE). For Immunohistochemistry, tissue sections of $5 \mu \mathrm{m}$ were deparaffinized and subjected to antigen retrieval by Proteinase $\mathrm{K}$ digestion (Roche) for $15 \mathrm{~min}$. Sections were stained for C3d with a polyclonal goat-anti-mouse antibody $(6.7 \mu \mathrm{g} / \mathrm{ml}$, catalog No. AF2655; R\&D Systems) or a normal goat IgG control antibody $(6.7 \mu \mathrm{g} / \mathrm{ml}$, catalog No. AB-108-C; R\&D Systems) followed by BrightVision poly-HRP anti-goat (Immunologic) and hematoxylin counterstain. Bright field photographs of slides were obtained and analyzed using a NanoZoomer RS slide scanner (NanoZoomer Digital Pathology System and NDP view software; Hamamatsu). No morphometric quantification was performed.

\section{Complement Component C3a ELISA}

The BALF was collected as described above for the cytokine ELISA measurements. The concentration of the complement activation product $\mathrm{C} 3 \mathrm{a}$ in BALF was measured in duplicates by a mouse-specific ELISA kit (E-EL-MO0337; Elabscience) following the manufacturer's recommendations.

\section{Statistical Analyses}

Data were analyzed using GraphPad Prism software, version 6. Comparisons between groups of WT and $\mathrm{KO}$ mice were made using the unpaired Student $t$ test and $\mathrm{p}<0.05$ was considered statistically significant.

\section{Results}

\section{Impaired Fungal Clearance in Ficolin KO Mice following A. fumigatus Infection}

To determine whether ficolins participate in host defense against $A$. fumigatus, groups of 10- to 12-week-old WT and ficolin $\mathrm{KO}$ mice were intranasally inoculated with a sublethal dose of $2 \times 10^{7} \mathrm{~A}$. fumigatus conidia. Mice were sacrificed $12-72 \mathrm{~h}$ p.i. for the quantification of fungal burden (CFU/lung) in whole lung homogenates (fig. 1a). The results showed that ficolin $\mathrm{KO}$ mice displayed statistically significant higher fungal loads than their WT counterparts at 24 h p.i., which demonstrates 


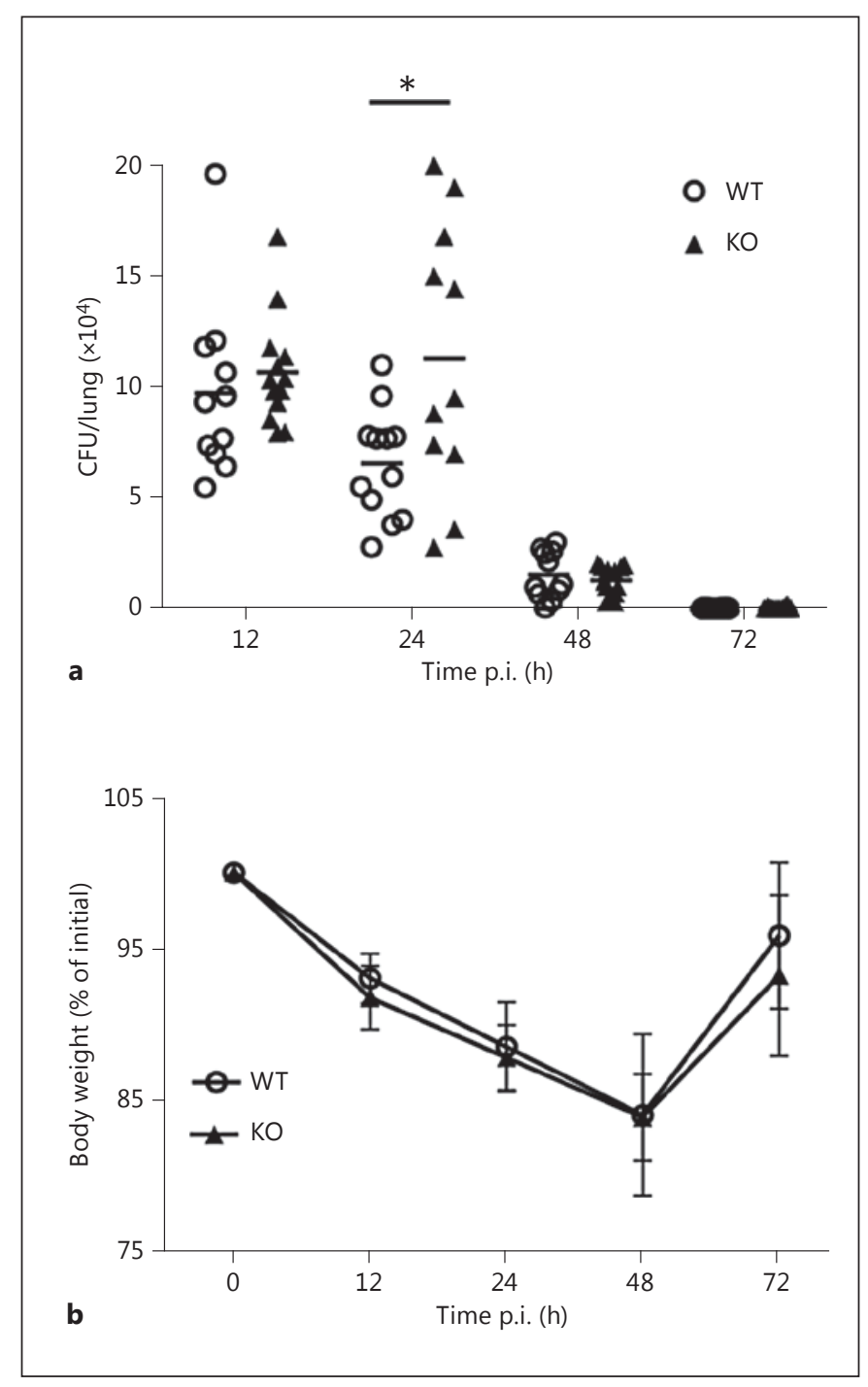

Fig. 1. Increased pulmonary fungal loads in ficolin-deficient mice $24 \mathrm{~h}$ after $A$. fumigatus infection. WT and ficolin $\mathrm{KO}$ mice were intranasally inoculated with $2 \times 10^{7} \mathrm{~A}$. fumigatus conidia. a Mice were sacrificed at the indicated time points for quantification of the fungal load (CFU/lung) in whole-lung homogenates. b Weight changes in mice during $A$. fumigatus infection. Results (mean \pm $\mathrm{SD})$ were obtained from two independent experiments $(\mathrm{n}=5-7 /$ group in each experiment). ${ }^{*} \mathrm{p}<0.05$ for ficolin $\mathrm{KO}$ to WT comparisons at each time point (unpaired two-tailed Student's t test).

that a lack of ficolins results in increased sensitivity to A. fumigatus infection. We found no fungal growth in the spleens of $A$. fumigatus-challenged mice 24 or 48 h p.i., which established that the infection did not disseminate from the lungs. This finding was expected because we utilized a nonlethal model of A. fumigatus infection. Accordingly, conidia were almost entirely cleared from the lungs (fig. 1a) and the body weight was regained (fig. 1b) in both ficolin $\mathrm{KO}$ and WT mice $72 \mathrm{~h}$ p.i. To elucidate whether both of the murine ficolins contribute to $\mathrm{A}$. $\mathrm{fu}$ migatus clearance, the fungal burden in ficolin-A KO mice, ficolin-B KO mice and WT mice was determined in subsequent experiments (online suppl. fig. 1; see www. karger.com/doi/10.1159/000447714 for all online suppl. material). Neither ficolin-A nor ficolin-B KO mice showed increased fungal loads over WT mice, which suggests a redundant role of the two murine forms of ficolins in the immune response to A. fumigatus.

\section{Pulmonary Cellular Infiltration of A. fumigatus- \\ Infected Mice}

Next, we tested whether the impaired fungal clearance observed in ficolin $\mathrm{KO}$ mice was associated with a reduced recruitment of inflammatory cells to the lungs. Mice were intranasally inoculated with A. fumigatus conidia or PBS as a control. After $24 \mathrm{~h}$ the lung tissues were collected for histology and stained with HE to assess the cellular infiltration and inflammation (fig. 2). Evaluation of the lung sections revealed the presence of inflammatory infiltrates in both WT and ficolin KO mice challenged with A. fumigatus (fig. 2b), and a comparable morphology of the two strains was observed. Normal lung histology was evident in sections from control mice mock infected with PBS (fig. 2a). In another experiment MPO levels were quantified in lung homogenates as a measure of neutrophil influx (fig. 3). The level of MPO in A. fumigatus-infected mice was increased $24 \mathrm{~h}$ p.i. compared to PBS mock-infected mice, confirming that neutrophils were recruited to the lungs following $A$. $f u$ migatus challenge. There was no statistically significant difference in MPO levels between WT and ficolin KO mice.

\section{Impaired Production of Proinflammatory Cytokines in Lungs of Ficolin KO Mice following A. fumigatus Infection}

To further evaluate the inflammatory response, we analyzed the lung proinflammatory cytokine profiles following A. fumigatus infection. WT and ficolin KO mice were intranasally inoculated with $A$. fumigatus conidia or $\mathrm{PBS}$ as a control. Mice were sacrificed $24 \mathrm{~h}$ p.i. to measure the mRNA expression of IL-1 $\beta$, IL- 6 and TNF- $\alpha$ in lung homogenates (fig. 4a). In addition, the expression of IL10 was measured; however, expression of this cytokine was not detected in any case. The results revealed that expression of IL- $1 \beta$ and IL- 6 was significantly decreased in ficolin $\mathrm{KO}$ mice compared to WT mice following 


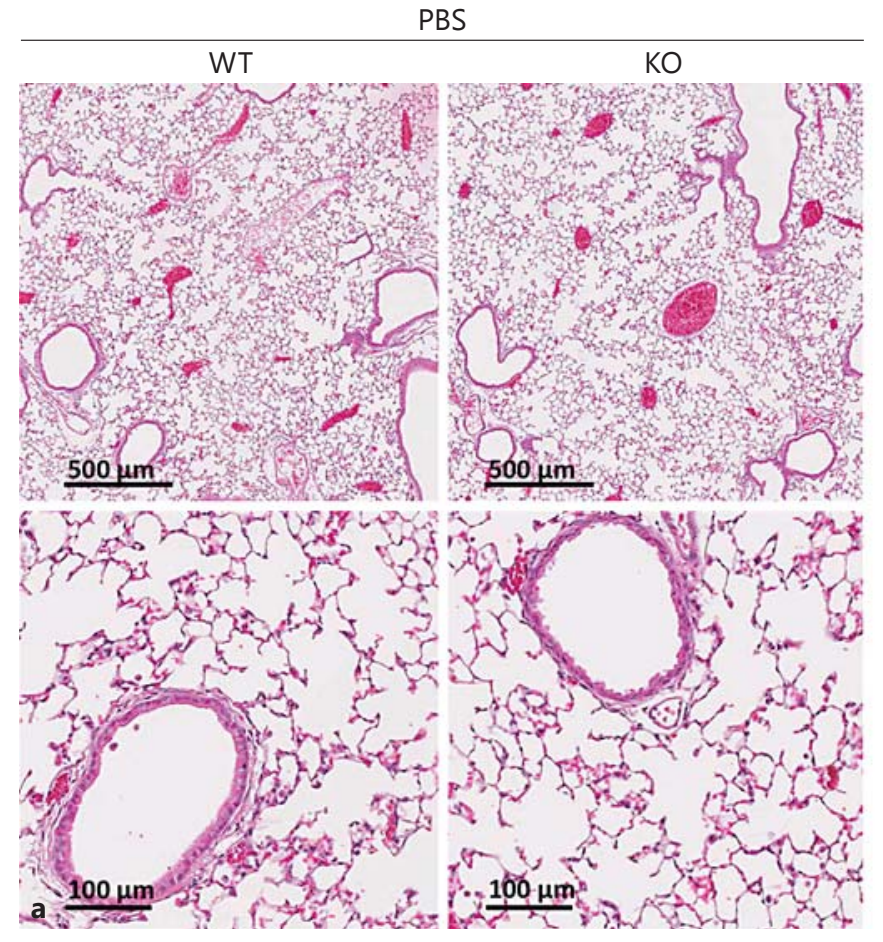

Fig. 2. Lung histology of mice following A. fumigatus infection. WT and ficolin $\mathrm{KO}$ mice were intranasally inoculated with PBS as controls (a) or $2 \times 10^{7} \mathrm{~A}$. fumigatus conidia (b) and sacrificed $24 \mathrm{~h}$ p.i. Lung tissues were harvested, fixed, and HE stained for

A. fumigatus infection. The difference in expression of TNF- $\alpha$ did not reach statistical significance. To support these findings, we measured the levels of the three cytokines in BALF $24 \mathrm{~h}$ p.i. in another set of experiments (fig. 4b). These results confirmed the mRNA findings; the protein levels of all three proinflammatory cytokines were decreased in ficolin $\mathrm{KO}$ mice compared to those in WT mice following A. fumigatus challenge, although only differences in IL-1 $\beta$ levels were statistically significant. The levels of all three cytokines in BALF from PBS mock-infected mice were below the detection limit in both strains. In addition, serum levels of the cytokines were measured; IL- $1 \beta$ and TNF- $\alpha$ were undetectable in the serum of both ficolin KO and WT mice following A. fumigatus challenge. In accordance with the lung cytokine levels, serum IL-6 was decreased in ficolin KO mice over WT mice, although the difference was not statistically significant (online suppl. fig. 2). Altogether, these results show a decreased production of proinflammatory cytokines in ficolin $\mathrm{KO}$ mice, which indicates that ficolins modulate the cytokine response following $A$. fumigatus infection.

Protective Role of Ficolins in Fungal Infection

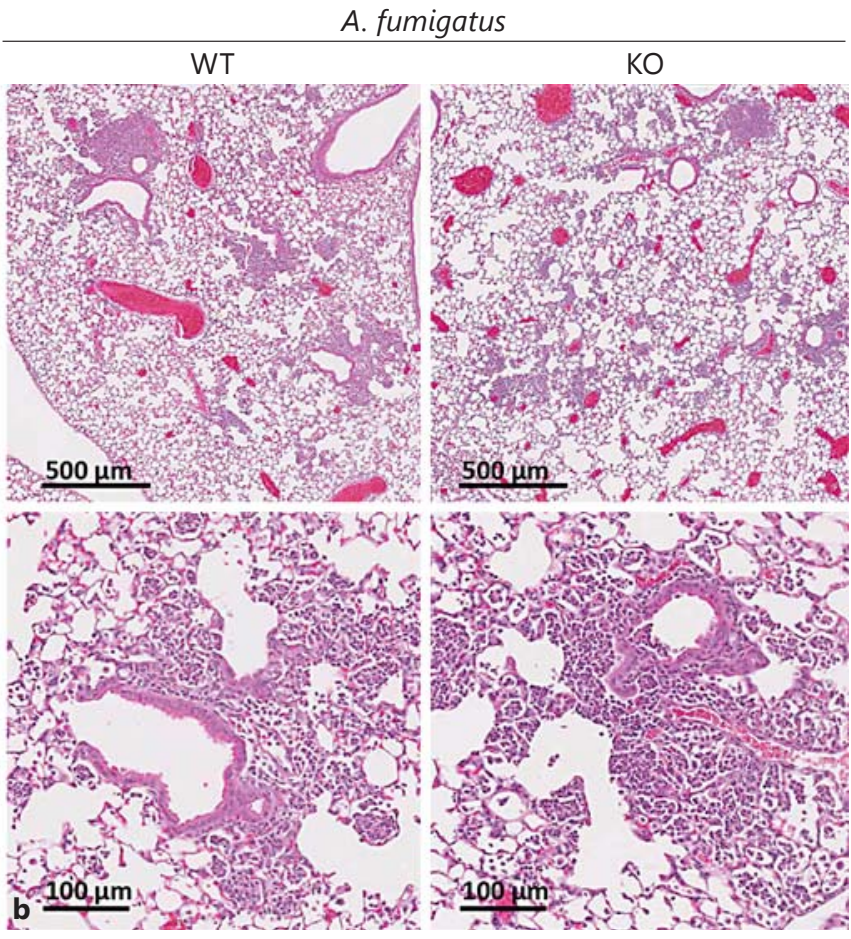

histological examination. Representative lung sections from WT or ficolin $\mathrm{KO}$ mice are shown. Images are representative of 2 mice in PBS mock-infected groups and 6 mice in the A. fumigatus-infected groups obtained from one experiment.

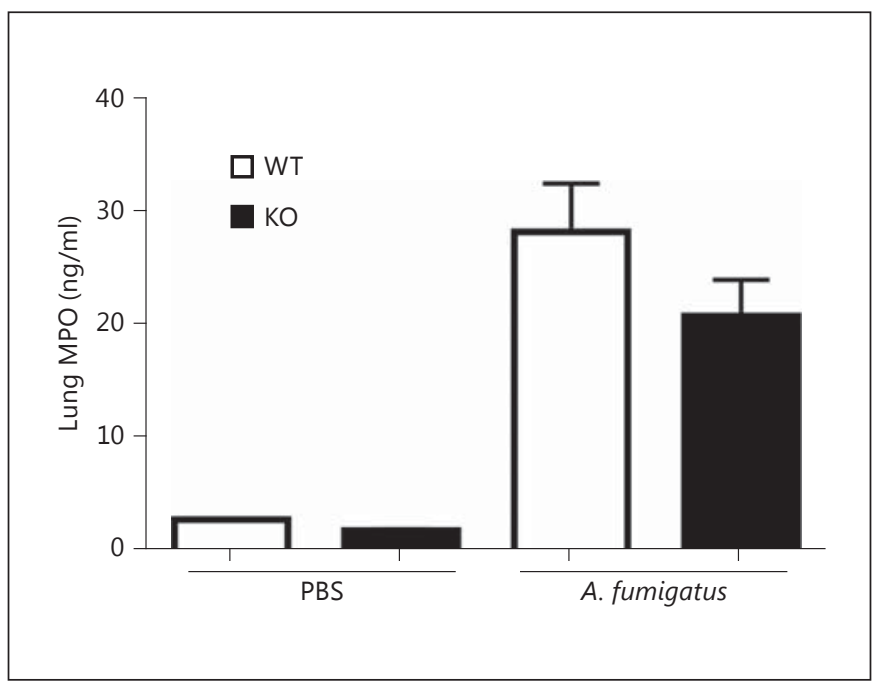

Fig. 3. Lung MPO concentration following A. fumigatus infection. WT and ficolin KO mice were intranasally inoculated with $2 \times 10^{7}$ A. fumigatus conidia and sacrificed $24 \mathrm{~h}$ p.i. Control mice received PBS. Lung tissues were harvested and the MPO concentration in supernatants of lung homogenates was measured by ELISA. Results represent means \pm SEM from one experiment with $n=2$ (PBS group) or $\mathrm{n}=6$ (A. fumigatus group).

J Innate Immun 2016;8:579-588 


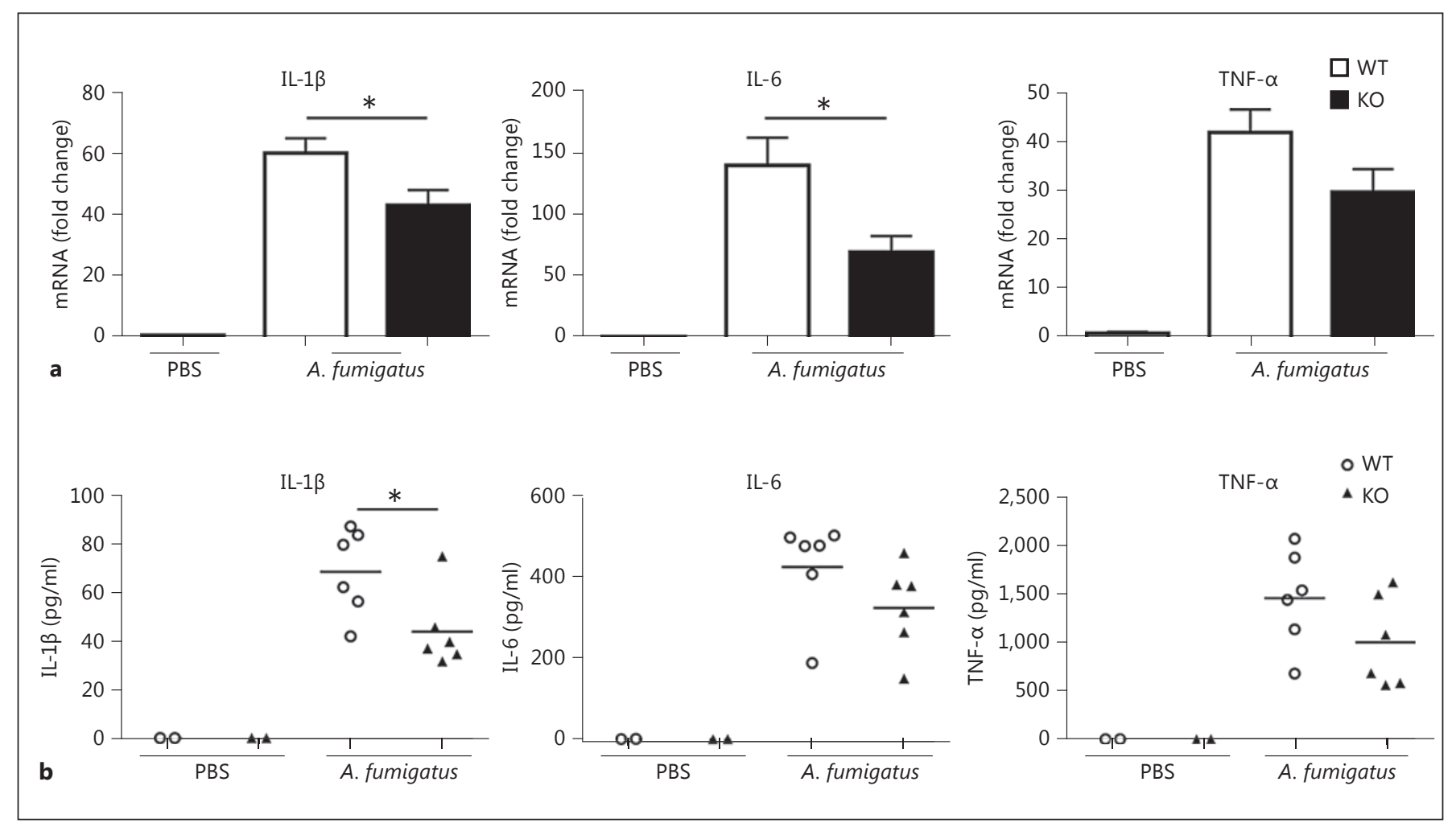

Fig. 4. Impaired production of proinflammatory cytokines in the lungs of ficolin-deficient mice following A. fumigatus infection. WT and ficolin KO mice were intranasally inoculated with $2 \times 10^{7}$ A. fumigatus conidia and sacrificed $24 \mathrm{~h}$ p.i. Control mice received PBS. a Lungs were removed and the expression of proinflammatory cytokines in lung homogenates was measured by qPCR; data represent means \pm SEM as the fold increase over the control group
(WT mock infected with PBS). b In separate experiments BALF was collected and cytokine concentrations in BALFs were measured by ELISA. The figures illustrate results from one experiment, representative of two independent experiments showing similar results, with $\mathrm{n}=2$ (PBS group) or $\mathrm{n}=6$ (A. fumigatus group) in each experiment. ${ }^{*} \mathrm{p}<0.05$ for ficolin $\mathrm{KO}$ to WT comparisons (unpaired two-tailed Student's t test).

\section{Unimpaired Complement Activation in Ficolin $\mathrm{KO}$}

\section{Mice following A. Fumigatus Infection}

Next, we sought to elucidate whether the impaired fungal clearance and cytokine induction in ficolin $\mathrm{KO}$ mice was due to a decreased activation of complement. Mice were intranasally inoculated with $A$. fumigatus conidia or PBS as a control; after $24 \mathrm{~h}$ the lung tissues were collected for immunohistochemistry and stained for the complement activation product C3d (the antibody also detects $\mathrm{C} 3 \mathrm{~b}$ and $\mathrm{C} 3 \mathrm{bi}$ ). Evaluation of lung sections revealed the deposition of $\mathrm{C} 3 \mathrm{~d}$ in mice challenged with A. fumigatus (fig. 5b, top panels); a similar level of C3d deposition was observed in ficolin KO and WT mice. No C3d was evident in lung sections from control mice mock infected with PBS (fig. 5a, top panels) or in sections stained with a control antibody (fig. $5 \mathrm{a}$, b, bottom panels). To support these findings, we quantitatively determined the levels of another complement cleavage product, C3a, in BALF (fig. 6). The results showed that C3a levels in the BALF of A. fumigatus-infected mice were increased compared to PBS mock-infected mice confirming that complement was activated following A. fumigatus infection. In agreement with the immunohistochemistry findings there was no difference between ficolin $\mathrm{KO}$ and WT mice, which implies that ficolins function independently of complement in the current infection model.

\section{Discussion}

Although ficolins have been identified as important pattern recognition molecules for bacterial and viral pathogens in vivo [20, 24-26], their role in fungal infection has not been established. Prior work from our research group has shown that human and murine ficolins bind to A. fumigatus in vitro $[27,28]$. In the present study, 


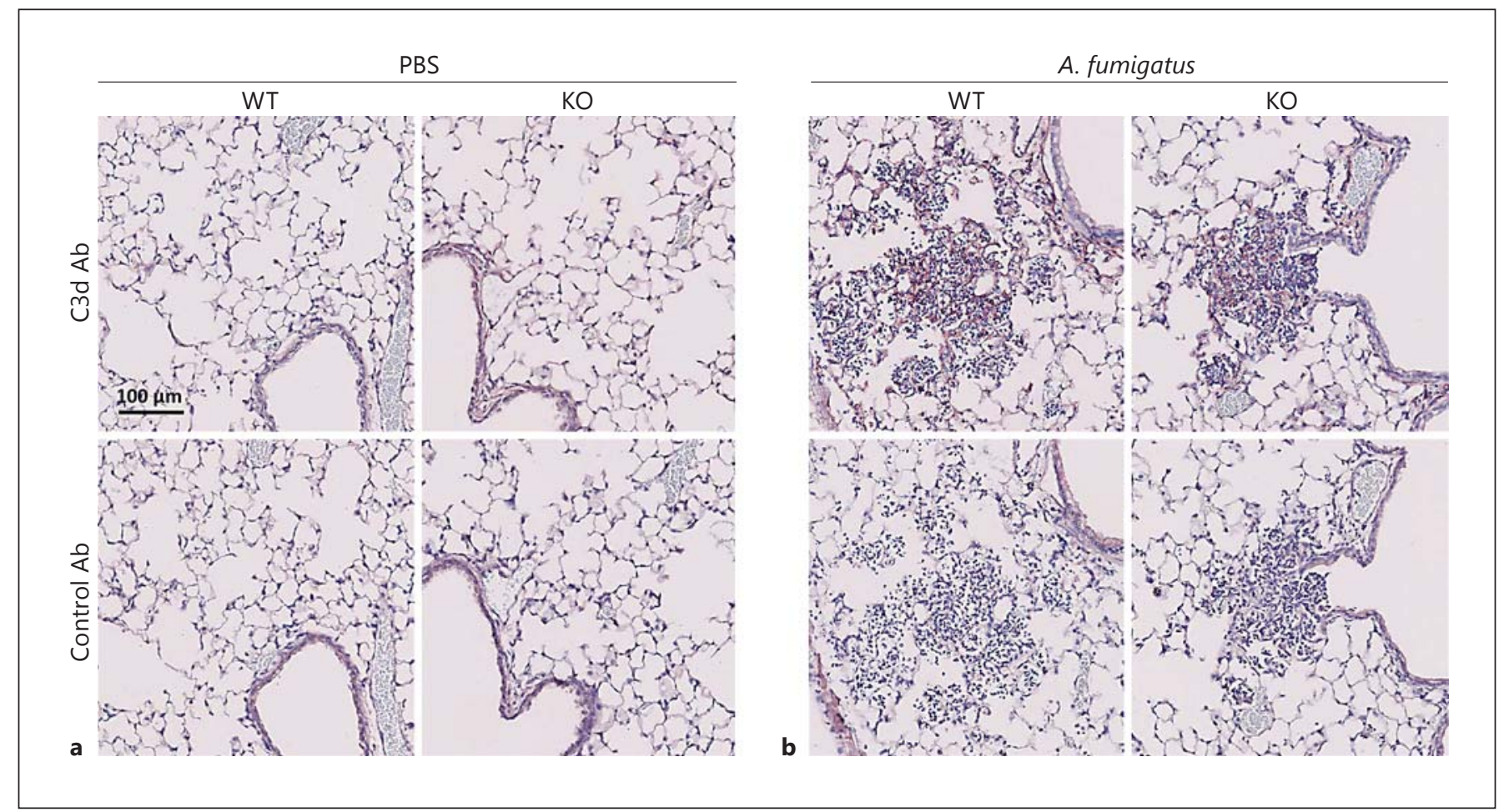

Fig. 5. Complement deposition in the lungs of mice following A. fumigatus infection. WT and ficolin KO mice were intranasally inoculated with PBS as controls (a) or $2 \times 10^{7}$ A. fumigatus conidia (b) and sacrificed $24 \mathrm{~h}$ p.i. Lungs were harvested, fixed, and immunohistochemically stained with an anti-C3d antibody (upper panels) or a control antibody (lower panels). Sections were counterstained with hematoxylin. Representative lung sections from $\mathrm{WT}$ or ficolin $\mathrm{KO}$ mice are shown. Images are representative of 2 mice in the PBS mock-infected group and 6 mice in the A. fumigatus-infected group obtained from one experiment. we identified a role for the ficolins in a mouse model of $A$. fumigatus infection. Two important points have emerged from this study. First, ficolins promote $A$. $f u$ migatus conidia clearance in the lungs. Second, ficolins modulate the cytokine production following A. fumigatus infection.

We found that fungal load was significantly higher in ficolin KO mice compared to WT mice $24 \mathrm{~h}$ p.i (fig. 1a). Recent studies showed that ficolin-A opsonization of $A$. fumigatus conidia enhanced the binding of conidia to A549 airway epithelial cells [29], human monocyte-derived macrophages and neutrophils [30]. Consequently, hyphal growth was inhibited and fungal killing was increased. Earlier studies also recognized that the human homologue of ficolin-A, ficolin-2, exhibits opsonic activity $[17,31]$. In the light of these data it seems likely that the delayed fungal clearance in the ficolin $\mathrm{KO}$ mice observed in our study could be credited to impaired opsonophagocytosis of the conidia. Interestingly, a model was proposed where pathogen-bound natural IgG:ficolin

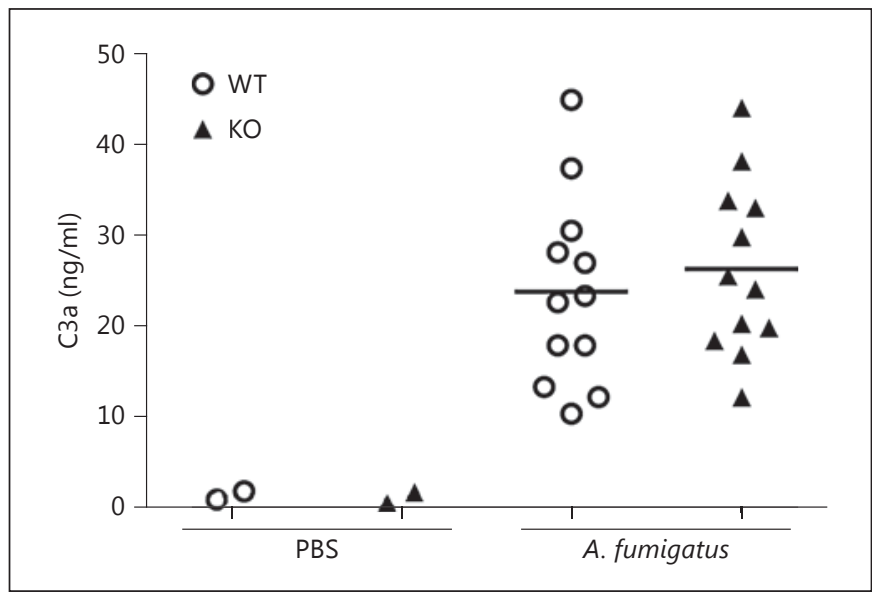

Fig. 6. Complement activation in lungs of mice following $A$. $f u$ migatus infection. WT or ficolin $\mathrm{KO}$ mice were intranasally inoculated with $2 \times 10^{7} \mathrm{~A}$. fumigatus conidia and sacrificed $24 \mathrm{~h}$ p.i. Control mice received PBS. The BALF was collected and C3a concentration in BALFs was measured by ELISA. Results were obtained from two experiments $(\mathrm{n}=4-8$ mice in the $A$. fumigatus groups per experiment). 
complexes enhance the uptake by phagocytes via $\mathrm{F} c \gamma \mathrm{R} 1$ independent of C3 [32]. Nevertheless, the precise mechanism by which ficolins promote fungal clearance in the lungs remains to be established. We found that $48 \mathrm{~h}$ after the onset of A. fumigatus infection the fungal loads were similar in ficolin KO and WT mice, which indicates that ficolins are of importance in the initial phase of infection. It is known that initial recognition of A. fumigatus conidia is managed by alveolar macrophages and pattern recognition receptors, whereas neutrophils are recruited subsequently [3]. The long pentraxin-3 (PTX3) is secreted by neutrophils and plays a pivotal role in protection against $A$. fumigatus in mice [33]. Hence, PTX3 constitutes an example of how redundant pathways might compensate in clearing conidia and modulating pulmonary inflammation over time. The objective of this study was to investigate the role of ficolins when fungal conidia are inhaled in the healthy host under normal circumstances. Therefore, we established a nonlethal model of infection in immunocompetent (ficolin $\mathrm{KO}$ ) mice. It is conceivable that the significance of ficolins is enhanced in the immunocompromised host, e.g. during neutropenic conditions. Indeed, it is in these situations that A. fumigatus represents a threat to human health [2]. Therefore, it is highly relevant to further elucidate the role of ficolins during fungal infections in an immunocompromised experimental model. Interestingly, a recent study detected the serum protein ficolin-2 in BALF from the lungs of patients with a diagnosis of invasive A. fumigatus infection [34], which highlights a clinical perspective for future studies.

Ficolin-A is a primarily liver-derived serum protein [35], whereas ficolin-B is present in serum only in very low concentrations [24]. It was recently shown that ficolin-B is stored in and released from immature granulocytic myeloid cells, and a role in the early infection-induced cellular response of these inflammatory cells was suggested [36]. We found that mice deficient in only ficolin-A or ficolin-B exhibited a similar clearance of conidia compared to WT mice (online suppl. fig. 1). These results imply that ficolin-A and ficolin-B play redundant roles in the clearance of $A$. fumigatus conidia. In contrast, Endo et al. [24] showed that the two murine ficolins did not work competitively in protection against $S$. pneumoniae; all of the three mouse strains, including ficolin-A KO, ficolin-B KO and double-deficient mice, displayed reduced survival and increased bacterial burdens in the lungs compared to WT mice. Taken together, it appears that the individual roles of the two murine ficolins depend on the pathogen and/or the biological setting.
We found that the observed delayed clearance of $A$. fumigatus conidia from the lungs in the absence of ficolins could not be attributed to impaired recruitment of inflammatory cells (fig. 2, 3). Actually, despite reduced production of proinflammatory cytokines in the lungs of A. fumigatus-infected ficolin KO mice, there was no significant difference between ficolin KO and WT mice regarding cellular recruitment to the lungs. These data indicate either that compensating mechanisms for prompting cellular recruitment exist, or that recruitment is independent of these cytokines. Another possibility is that the difference in cellular recruitment between ficolin $\mathrm{KO}$ and WT mice was too small to detect with the employed methods.

In our study, A. fumigatus-induced production of proinflammatory cytokines was reduced by approximately $30 \%$ in ficolin KO mice compared to WT mice (fig. 4). Similarly, a proinflammatory role of MBL in a mouse model of $A$. fumigatus infection has been suggested [9]. Consistent with our data, recent studies demonstrated the ability of ficolins to modulate the inflammatory response. Specifically, it was shown that ficolin-2 stimulated murine macrophages to produce IFN- $\gamma$, IL-17A, TNF- $\alpha$ and IL-6 in vitro, and that ficolin- $\mathrm{A} \mathrm{KO}$ mice exhibited decreased production of TNF- $\alpha$ and IL-17A in vivo upon stimulation with LPS [26]. Other investigators reported that ficolin-A increased the production of IL-8 from human lung epithelial cells following A. fumigatus challenge in vitro [29]. On the other hand, the same group showed that ficolin-A or ficolin-2 opsonization of $A$. fumigatus leads to decreased levels of IL-8, IL- $1 \beta$, IL-6, and TNF- $\alpha$ from human monocyte-derived macrophages and neutrophils $[30,34]$. However, the behavior of these cells in vitro might not represent all in vivo functions that are likely influenced by paracrine signals. Cytokines trigger a strong localized inflammatory response required for the hazard elimination, but the response must be tightly regulated to prevent excessive tissue-damaging inflammation. In the setting of overwhelming inflammation, signaling pathways might contribute to a poor outcome. In this study, we have identified a role for ficolins as cytokine modulators. In addition to the opsonizing activity, ficolins are known to activate the lectin pathway of complement via interaction with MBL-ficolin-associated serine proteases [12], a property they share with MBL. Consequently, one mechanism by which ficolins could increase the cytokine response is through activation of complement. However, our results revealed that the complement activation was unimpaired in the ficolin $\mathrm{KO}$ mice, which indicates that complement-independent mechanisms must be involved 
(fig. 5, 6). Interestingly, it has been shown that MBL promote IL- 6 and TNF- $\alpha$ production, not through its capacity to act as an opsonin or to activate complement, but via a cooperation with TLR2/6 $[37,38]$. A similar role for ficolins could be hypothesized based on our findings.

As stated above, we found that the absence of ficolins did not reduce complement activation in vivo following A. fumigatus infection (fig. 5, 6). In addition, we found no difference between WT and ficolin KO serum regarding $\mathrm{C} 3$ or $\mathrm{C} 4$ deposition on $A$. fumigatus in vitro (online suppl. fig. 3). These results are consistent with another study where the contribution of ficolins to complement activation on A. fumigatus was investigated. The investigators used WT, ficolin-A- or MBL-deficient mouse sera and reported that ficolin-A-bound A. fumigatus conidia did not activate the lectin complement pathway, but that complement deposition was mediated via binding of MBL-C [29]. In addition, PTX3 has been shown to activate complement on A. fumigatus [39]. Therefore, it is likely that the potential contribution of ficolins to complement activation on A. fumigatus is negligible in the presence of other complement activators. In contrast, it was reported that ficolin $\mathrm{KO}$ serum showed lower complement deposition on S. pneumoniae compared to WT serum [24, 25]. Importantly, MBL-deficient serum showed the same level of deposition as WT serum, which indicates that MBL did not drive lectin pathway activation on S. pneumoniae [25]. Hence, although MBLs are the major initiators of the lectin pathway, the data suggested that MBLs are not involved in S. pneumoniae infection, which might explain the difference to (lack of) ficolin-mediated complement activation on A. fumigatus. It should be noted that the ficolin-mediated lectin pathway appears to be more important in humans than in mice; in humans, the serum concentrations of ficolins are several times greater than MBL [13], whereas the opposite is the case for mice [40].

We conclude that ficolins promote the clearance of A. fumigatus and modulate the inflammatory cytokine response independent of complement activation. These results demonstrate that ficolins participate in host defense against $A$. fumigatus infections in vivo. The study provides supporting evidence to the role of ficolins demonstrated in vitro and extend our knowledge of protection against $A$. fumigatus infection.

\section{Acknowledgements}

We want to express our gratitude to Dr. Endo, Fukushima University, Japan, for providing us with ficolin-A and ficolin-B knockout mice. Furthermore, we want to thank Ms. Vibeke Witved for her excellent technical and administrative assistance.

\section{References}

1 Brown GD, Denning DW, Gow NAR, Levitz SM, Netea MG, White TC: Hidden killers: human fungal infections. Sci Transl Med 2012;4: $165 \mathrm{rv} 13$.

-2 Segal BH: Aspergillosis. N Engl J Med 2009; 360:1870-1884

3 Margalit A, Kavanagh K: The innate immune response to Aspergillus fumigatus at the alveolar surface. FEMS Microbiol Rev 2015;39: 670-687.

4 Carvalho A, De Luca A, Bozza S, Cunha C, D'Angelo C, Moretti S, et al: TLR3 essentially promotes protective class I-restricted memory CD8 ${ }^{+} \mathrm{T}$-cell responses to Aspergillus fumigatus in hematopoietic transplanted patients. Blood 2012;119:967-977.

5 Ramaprakash H, Ito T, Standiford TJ, Kunkel SL, Hogaboam CM: Toll-like receptor 9 modulates immune responses to Aspergillus fumigatus conidia in immunodeficient and allergic mice. Infect Immun 2009;77:108119.

6 Netea MG, Warris A, van der Meer JWM, Fenton MJ, Verver-Janssen TJG, Jacobs LEH, et al: Aspergillus fumigatus evades immune recognition during germination through loss of toll-like receptor-4-mediated signal transduction. J Infect Dis 2003;188:320-326.

-7 Bellocchio S, Moretti S, Perruccio K, Fallarino F, Bozza S, Montagnoli C, et al: TLRs govern neutrophil activity in aspergillosis. J Immunol 2004;173:7406-7415.

\$8 Werner JL, Metz AE, Horn D, Schoeb TR, Hewitt MM, Schwiebert LM, et al: Requisite role for the dectin- $1 \beta$-glucan receptor in pulmonary defense against Aspergillus fumigatus. J Immunol 2009;182:4938-4946.

-9 Kaur S, Gupta VK, Thiel S, Sarma PU, Madan $\mathrm{T}$ : Protective role of mannan-binding lectin in a murine model of invasive pulmonary aspergillosis. Clin Exp Immunol 2007;148:382389.

10 Madan T, Reid KBM, Clark H, Singh M, Nayak A, Sarma PU, et al: Susceptibility of mice genetically deficient in SP-A or SP-D gene to invasive pulmonary Aspergillosis. Mol Immunol 2010;47:1923-1930.

-11 Garlanda C, Hirsch E, Bozza S, Salustri A, De Acetis M, Nota R, et al: Non-redundant role of the long pentraxin PTX3 in anti-fungal innate immune response. Nature 2002;420: 182-186.
12 Endo Y, Matsushita M, Fujita T: New insights into the role of ficolins in the lectin pathway of innate immunity. Int Rev Cell Mol Biol 2015;316:49-110.

13 Garred P, Honoré C, Ma YJ, Rørvig S, Cowland J, Borregaard N, et al: The genetics of ficolins. J Innate Immun 2010;2:3-16.

14 Matsushita M, Endo Y, Fujita T: Cutting edge: complement-activating complex of ficolin and mannose-binding lectin-associated serine protease. J Immunol 2000; 164:2281-2284.

15 Ricklin D, Hajishengallis G, Yang K, Lambris JD: Complement: a key system for immune surveillance and homeostasis. Nat Immunol 2010;11:785-797.

16 Endo Y, Liu Y, Kanno K, Takahashi M, Matsushita M, Fujita T: Identification of the mouse $\mathrm{H}$-ficolin gene as a pseudogene and orthology between mouse ficolins $\mathrm{A} / \mathrm{B}$ and human L-/M-ficolins. Genomics 2004;84:737744.

17 Matsushita M, Endo Y, Taira S, Sato Y, Fujita $\mathrm{T}$, Ichikawa $\mathrm{N}$, et al: A novel human serum lectin with collagen- and fibrinogen-like domains that functions as an opsonin. J Biol Chem 1996;271:2448-2454.
Protective Role of Ficolins in Fungal Infection
J Innate Immun 2016;8:579-588 DOI: $10.1159 / 000447714$ 
18 Kjaer TR, Hansen AG, Sørensen UBS, Holm AT, Sørensen GL, Jensenius JC, et al: M-ficolin binds selectively to the capsular polysaccharides of Streptococcus pneumoniae serotypes 19B and 19C and of a Streptococcus mitis strain. Infect Immun 2013;81:452-459.

-19 Krarup A, Sørensen UBS, Matsushita M, Jensenius JC, Thiel S: Effect of capsulation of opportunistic pathogenic bacteria on binding of the pattern recognition molecules mannanbinding lectin, L-ficolin, and $\mathrm{H}$-ficolin. Infect Immun 2005;73:1052-1060.

20 Pan Q, Chen H, Wang F, Jeza VT, Hou W, Zhao Y, et al: L-ficolin binds to the glycoproteins hemagglutinin and neuraminidase and inhibits influenza A virus infection both in vitro and in vivo. J Innate Immun 2012;4:312324.

21 Munthe-Fog L, Hummelshoj T, Honoré C, Moller ME, Skjoedt MO, Palsgaard I, et al: Variation in FCN1 affects biosynthesis of ficolin- 1 and is associated with outcome of systemic inflammation. Genes Immun 2012;13: 515-522.

22 Cedzynski M, Atkinson APM, St Swierzko A, MacDonald SL, Szala A, Zeman K, et al: L-ficolin (ficolin-2) insufficiency is associated with combined allergic and infectious respiratory disease in children. Mol Immunol 2009; 47:415-419.

-23 Munthe-Fog L, Hummelshøj T, Honoré C, Madsen HO, Permin H, Garred P: Immunodeficiency associated with FCN3 mutation and ficolin-3 deficiency. N Engl J Med 2009; 360:2637-2644.

-24 Endo Y, Takahashi M, Iwaki D, Ishida Y, Nakazawa N, Kodama T, et al: Mice deficient in ficolin, a lectin complement pathway recognition molecule, are susceptible to Streptococcus pneumoniae infection. J Immunol 2012;189: 5860-5866.
25 Ali YM, Lynch NJ, Haleem KS, Fujita T, Endo $\mathrm{Y}$, Hansen S, et al: The lectin pathway of complement activation is a critical component of the innate immune response to pneumococcal infection. PLoS Pathog 2012;8:e1002793.

26 Luo F, Sun X, Wang Y, Wang Q, Wu Y, Pan $\mathrm{Q}$, et al: Ficolin-2 defends against virulent Mycobacteria tuberculosis infection in vivo, and its insufficiency is associated with infection in humans. PLoS One 2013;8:e73859.

27 Ma YJ, Doni A, Hummelshøj T, Honoré C, Bastone A, Mantovani A, et al: Synergy between ficolin-2 and pentraxin 3 boosts innate immune recognition and complement deposition. J Biol Chem 2009;284:28263-28275.

28 Hummelshøj T, Ma YJ, Munthe-Fog L, Bjarnsholt T, Moser C, Skjoedt M-O, et al: The interaction pattern of murine serum ficolin-A with microorganisms. PLoS One 2012;7:e38196.

29 Bidula S, Kenawy H, Ali YM, Sexton D, Schwaeble WJ, Schelenz S: Role of ficolin-A and lectin complement pathway in the innate defense against pathogenic Aspergillus species. Infect Immun 2013;81:1730-1740.

30 Bidula S, Sexton DW, Schelenz S: Serum opsonin ficolin-A enhances host-fungal interactions and modulates cytokine expression from human monocyte-derived macrophages and neutrophils following Aspergillus fumigatus challenge. Med Microbiol Immunol 2016; 205:133-142.

$>31$ Fujieda M, Aoyagi Y, Matsubara K, Takeuchi Y, Fujimaki W, Matsushita M, et al: L-ficolin and capsular polysaccharide-specific IgG in cord serum contribute synergistically to opsonophagocytic killing of serotype III and V group B streptococci. Infect Immun 2012;80: 2053-2060.

32 Panda S, Zhang J, Tan NS, Ho B, Ding JL: Natural IgG antibodies provide innate protection against ficolin-opsonized bacteria. EMBO J 2013;32:2905-2919.
33 Garlanda C, Hirsch E, Bozza S, Salustri A, De Acetis M, Nota R, et al: Non-redundant role of the long pentraxin PTX3 in anti-fungal innate immune response. Nature 2002;420: 182-186.

34 Bidula S, Sexton DW, Abdolrasouli A, Shah A, Reed A, Armstrong-James D, et al: The serum opsonin L-ficolin is detected in lungs of human transplant recipients following fungal infections and modulates inflammation and killing of Aspergillus fumigatus. J Infect Dis 2015;212:234-246.

35 Fujimori Y, Harumiya S, Fukumoto Y, Miura Y, Yagasaki K, Tachikawa H, et al: Molecular cloning and characterization of mouse ficolin-A. Biochem Biophys Res Commun 1998; 244:796-800.

36 Weber-Steffens D, Hunold K, Kürschner J, Martinez SG, Elumalai P, Schmidt D, et al: Immature mouse granulocytic myeloid cells are characterized by production of ficolin-B. Mol Immunol 2013;56:488-496.

37 Shi L, Takahashi K, Dundee J, Shahroor-Karni S, Thiel S, Jensenius JC, et al: Mannosebinding lectin-deficient mice are susceptible to infection with Staphylococcus aureus. J Exp Med 2004;199:1379-1390.

38 Ip WKE, Takahashi K, Moore KJ, Stuart LM, Ezekowitz RAB: Mannose-binding lectin enhances Toll-like receptors 2 and 6 signaling from the phagosome. J Exp Med 2008;205: 169-181.

39 Moalli F, Doni A, Deban L, Zelante T, Zagarella S, Bottazzi B, et al: Role of complement and $\mathrm{FCY}_{\mathrm{C}}$ receptors in the protective activity of the long pentraxin PTX3 against Aspergillus fumigatus. Blood 2010;116:5170-5180.

40 Hansen S, Thiel S, Willis A, Holmskov U, Jensenius JC: Purification and characterization of two mannan-binding lectins from mouse serum. J Immunol 2000;164:2610-2618. 